\title{
The Clinical Picture and the Course of Rosacea Depending on the Presence of Demodex Mite and its Species Identity
}

\author{
Kubanov A, Gallyamova Y*, and Grevtseva A \\ Russian Medical Academy of Continuous Professional Education of the Ministry of \\ Healthcare of the Russian Federation, Russia
}

*Corresponding author: Yulia Gallyamova, 2/1, building 1, Barrikadnaya street, Moscow, 125993, Russia, Tel: +79161704546; E-mail: derma2006@yandex.ru

\section{Research Article \\ Volume 2 Issue 2}

Received Date: March 09, 2017

Published Date: June 06, 2017

DOI: $10.23880 /$ cdoaj 16000123

\section{Abstract}

The article presents the analysis of the clinical picture of patients with rosacea of different degrees of severity complicated with demodicosis. The dermatovenereology life quality index was evaluated for patients with rosacea in the presence and absence of Demodex mites. Presence of Demodex mites reduces the life quality of patients with rosacea. The duration of pathological processes on the facial skin, as well as the number of relapses were assessed. The course of rosacea was analyzed depending on the species identity of Demodex mites (Demodex folliculorum longus and Demodex folliculorum brevis). In the structure of rosacea diagnoses Demodex folliculorum longus is significantly more prevalent ( $\mathrm{n}$ $=20 ; 66.7 \%$ ) then Demodex folliculorum brevis ( $n=8 ; 26.7)$.

Keywords: Rosacea; Dermatosis; Demodicosis; Demodex mites

\section{Introduction}

Rosacea is a chronic dermatosis characterized by lesions of the skin in the form of erythema and papulopustules elements. It affects mainly people at the age of $30-50$ years. $14 \%$ of women and $5 \%$ of men with light skin phototype are subject to this type of dermatosis [1].

Thus, rosacea is a very acute problem because of its prevalence among the population. In terms of rosacea causes, a certain part is assigned to the parasitic theory [2]. According to this theory, the formation of papules and pustules on the facial skin contributes to the follicular mite (Demodex). The mite belongs to Demodex genus, Demodicidae family, suborder Trombidiformes, class Acariformes. For the first time the mite was revealed by Berger $\mathrm{F}$ in earwax of acoustic meatus of a person in 1841, the same year Henle FGJ revealed the mite on human skin. A year after, in 1842 Simon G established the existence of the parasite in hair follicles and for the first time described the morphological properties, calling them Acarus folliculorum (from Greek - "folliculorum mites"). Later Simon G (1842) and Owen R (1843) attributed the found mites to genus Demodex. Much later, after more than half a century English acarologist Hirst S (19171923) identified 21 species and several subspecies of Demodex mites in animals [3]. Later, studying mite parasitizing on human skin, Akbulatova LH (1970) discovered and described two forms: Demodex folliculorum longus and Demodex folliculorum brevis [4]. Demodex mites are equally spread among all races and all age groups [5]. Only rare cases of mite detection at newborns were described [6] and as a whole a low level of dissemination is registered in children. In all probability, this is due to lower production of sebum in children as compared to adults [7]. Analyzing the occurrence frequency of Demodex mites depending on 


\section{Clinical Dermatology Open Access Journal}

their specific accessory (Demodex folliculorum longus, Demodex folliculorum brevis), according to literature, it was revealed that Demodex folliculorum longus is detected more frequently than Demodex folliculorum brevis $[2,8]$.

As many researches show, even if there are some mites on the skin, the clinical picture does not always develop. On this basis, it can be argued that Demodex mites are opportunistic parasites [5]. So far the exact reasons resulting in pathogenicity of Demodex mites are not determined, the existing theories are diverse and contradictory [9].

Considering the relevance of this issue, the authors have conducted the survey of patients with rosacea complicated and uncomplicated with demodicosis and healthy volunteers.

The goal of the research was to assess the clinical picture, severity and courses of rosacea, complicated and uncomplicated with Demodex.

\section{Materials and methods}

There were 30 patients with rosacea complicated with demodicosis (group I), 30 patients with rosacea without demodicosis (group II) before treatment and 30 healthy volunteers (group III) under the supervision. Rosacea was diagnosed during the primary outpatient admission based on the clinical picture.

The study group included patients with the following criteria:

1. Men and women with acne and rosacea

2. Age 18 years or older

3. Informed consent of patients to participate in the study.

Exclusion criteria from the study:

1. Existence of concurrent somatic diseases of a heavy current or neoplastic character

2. Existence of hyperandrogenism

3. Alcohol or drug addiction

4. Lack of desire at the patient to continue the study

5. The occurrence of allergic reactions and the development of significant side effects during treatment;

6. Pregnancy and lactation.

All patients included in groups I and II have answered the questions of the questionnaire "Dermatology Life Quality Index," which consists of 10 points. Each response is scored on a scale from 0 to 3 . Then the scores were summed up and the result was compared between the patients of groups I and II.

The degree of rosacea severity was assessed according to the classification of the National Rosacea Society (2002):

Subtypes of rosacea:

Subtype I - erythematotelangiectatic

Subtype II - papulopustules

Subtype III - phymatous

Subtype IV - ocular [10].

This classification has been modified slightly, due to the lack of ophthalmorosacea cases in our practice.

Definition of existence and species identity of Demodex mites was performed by microscopy of skin scrapes, contents of sebaceous glands, hair follicles of eyelashes and/or eyebrows, calculation of the found individuals per unit area $\left(1 \mathrm{~cm}^{2}\right)$. The material was taken from the places of the greatest congestion of sebaceous glands on the face - nose wings, chin, glabellar area by means of scraping, pulling out eyebrows and eyelashes with tweezers. Microscopic examination was performed with compulsory definition of species identity of Demodex mites (Demodex folliculorum longus, Demodex folliculorum brevis) and quantitative load per unit area $\left(1 \mathrm{~cm}^{2}\right)$. Microscope magnification was $\mathrm{x} 10$ and $\mathrm{x} 40$. Patients with quantitative load of more than 5 adult mites at $1 \mathrm{~cm}^{2}$ and clinical picture of rosacea made the first group.

Statistical data processing was carried out with the use of Microsoft Excel 2013 and SPSS 21 software. The relationship of categorical indicators was established using Fisher's exact test. Fisher's exact test is a test of statistical significance used in the analysis of categorical data when sample sizes are small. To assess the significance of differences in the absence of normal distribution Mann-Whitney criteria and Kruskal-Wallis test for multiple comparisons were applied. Differences were considered significant at $\mathrm{p}<0.05$.

\section{Results}

In total 90 patients at the age of 18 - 79 (average age $30.0 \pm 11.9$ ) were under the supervision, including 39 male and 51 female patient. The diagnosis of rosacea has been exposed to 60 patients ( 30 patients in group I and 30 in group II).

According to the method of study, patients were divided into two groups. Group I included patients with 


\section{Clinical Dermatology Open Access Journal}

Demodex mites, which were detected by the method of microscopy scrapings in number of more than 5 individuals on $1 \mathrm{~cm}^{2}$, group II consisted of patients with a negative test for the presence of Demodex mites (Table 1).

\begin{tabular}{|c|c|c|c|}
\hline \multicolumn{3}{|c|}{$\begin{array}{c}\text { I group (with the presence of } \\
\text { Demodex mites) }\end{array}$} & $\begin{array}{c}\text { Total } \\
\text { (n; \%) }\end{array}$ \\
\hline \multirow{2}{*}{ Gender } & Men & Women & \multirow{2}{*}{$30(100 \%)$} \\
\cline { 2 - 3 } & $\mathrm{n}=12 ; 40.0 \%$ & $\mathrm{n}=18 ; 60.0 \%$ & \\
\hline Age & $44 \pm 10.4$ & $51 \pm 17.6$ & \multirow{2}{*}{$30(100 \%)$} \\
\hline \multirow{2}{*}{ II group (with the absence of } \\
Demodex mites)
\end{tabular}

Table 1: Distribution of patients by gender, age, diagnosis and the presence of Demodex mites.

Group I included 30 people with the diagnosis of "Rosacea" having an average age of 47 years ( $\min =32$, max $=79$ ). Group II included 30 people with the diagnosis of "Rosacea" having an average age of 45 years $(\mathrm{min}=27$, $\max =58$ ).

Analysis of the data duration of rosacea in the groups was as follows (Table 2):

\begin{tabular}{|c|c|c|c|}
\hline Disease duration & $\begin{array}{c}\text { Group I } \\
\text { (n; \%) }\end{array}$ & $\begin{array}{c}\text { Group II } \\
\text { (n; \%) }\end{array}$ & $\begin{array}{c}\text { Total } \\
\text { (n; \%) }\end{array}$ \\
\hline Less than 1 year & $3 ; 5$ & $12 ; 20^{*}$ & $15 ; 25$ \\
\hline 1 - 5 years & $5 ; 8.3$ & $12 ; 20^{*}$ & $17 ; 28.3$ \\
\hline More than 5 years & $22 ; 36.7^{*}$ & $6 ; 10$ & $28 ; 46.7$ \\
\hline Total & $30 ; 50$ & $30 ; 50$ & $60 ; 100$ \\
\hline
\end{tabular}

Table 2: Duration of rosacea in patients of groups I and II.

$*$ - $\mathrm{p}<0.01$

The obtained data show that there are statistically significant differences $(p<0.01)$ in patients with rosacea who constituted groups I and II, that is, the presence of Demodex mites in rosacea contributes to a longer course of the disease.

Patients who turned repeatedly to the attendance were surveyed for the presence of rosacea recurrence within a year. By the number of relapses per year, they were divided into three groups: with one relapse per year, with relapses from one to three times and with relapses more than three times per year (Table 3).

\begin{tabular}{|c|c|c|c|c|}
\hline & $\begin{array}{c}\mathbf{1} \\
\text { relapse } \\
\text { (n; \%) }\end{array}$ & $\begin{array}{c}\mathbf{1 - 3} \\
\text { relapses } \\
\text { (n; \%) }\end{array}$ & $\begin{array}{c}\mathbf{3} \text { and more } \\
\text { relapses } \\
\text { (n; \%) }\end{array}$ & $\begin{array}{c}\text { Total } \\
\text { (n; \%) }\end{array}$ \\
\hline Group I & $3 ; 7.9$ & $15 ; 39.5^{*}$ & $4 ; 10.5$ & $22 ; 57.9$ \\
\hline Group II & $10 ; 26.3^{*}$ & $4 ; 10.5$ & $2 ; 5.3$ & $16 ; 42.1$ \\
\hline Total & $13 ; 34.2$ & $19 ; 50$ & $6 ; 15.8$ & $38 ; 100$ \\
\hline
\end{tabular}

Table 3: The number of rosacea relapses in patients of groups I and II within a year.

$*$ - $\mathrm{p}<0.05$

The DLQI is the method for assessing the impact of dermatological diseases on the psycho-emotional state of a person. This questionnaire was developed in 1990-1994 by the scientists of the Department of Dermatology of the University of Cardiff (Cardiff University, UK) Andrew Finlay Y and Gul Karim Khan [11].

When comparing average values of the DLQI, a statistically reliable difference was revealed. In patients of group I the average value of the DLQI amounted to $12.5 \pm$ $4.5(\min =5.0 ; \max =19.0)$. This indicator shows that the disease has a very strong influence on the life of a patient. In patients of group II the average value of the DLQI was equal to $12.5 \pm 4.5(\min =5.0 ; \max =19.0)$, which means that the disease has moderate influence on the life of patients $(\mathrm{p}<0.05)$.

Comparing indicators of the DLQI of patients in group I - between the carriers of Demodex folliculorum longus and Demodex folliculorum brevis, as well as the presence of parasitism by both species of mites, the following data were obtained; they are given in table 4 .

\begin{tabular}{|c|c|c|c|c|}
\hline Species accessory & $\begin{array}{c}\text { Number of } \\
\text { patients }\end{array}$ & $\begin{array}{c}\text { Average } \\
\text { value of } \\
\text { DLQI }\end{array}$ & Min & Max \\
\hline $\begin{array}{c}\text { Demodex } \\
\text { folliculorum longus }\end{array}$ & 18 & $15.5^{*}$ & 5 & 19 \\
\hline $\begin{array}{c}\text { Demodex } \\
\text { folliculorum brevis }\end{array}$ & 8 & $10.5^{*}$ & 9 & 13 \\
\hline $\begin{array}{c}\text { Combined cases } \\
\text { (Demodex } \\
\text { folliculorum longus } \\
+\end{array}$ & 4 & 13.5 & 13 & 14 \\
$\begin{array}{c}\text { Demodex } \\
\text { folliculorum } \\
\text { brevis) }\end{array}$ & & & & \\
\hline
\end{tabular}

Table 4: The DLQI of patients with rosacea in group I depending on the species identity of Demodex mites

$*$ - $\mathrm{p}<0.05$

Table 4 shows that rosacea complicated by the presence of Demodex folliculorum brevis has a moderate impact on the patients' lives (average value of 


\section{Clinical Dermatology Open Access Journal}

dermatology life quality index is 10.5 ). In the presence of Demodex folliculorum longus and combined cases of simultaneous detection of two species of mites, the disease greatly affects the patients' lives (average value of DLQI - 15.5 and 13.5, respectively). DLQI of patients with rosacea included in group I with the presence of Demodex folliculorum longus and Demodex folliculorum brevis are statistically significantly different $(\mathrm{p}<0.05)$, which is logically natural, since it was found that Demodex folliculorum longus is detected at patients with more severe forms of the disease. Statistical differences between patients having Demodex folliculorum longus,
Demodex folliculorum brevis and combined cases of parasitism of both species have not been identified.

Local status was described during the initial reception of patients. The pathological process of the skin was evaluated on the face, after which the data were entered in the form of primary documentation.

Figures 1-6 show patients diagnosed with rosacea, complicated and uncomplicated with demodicosis, included in groups I and II.

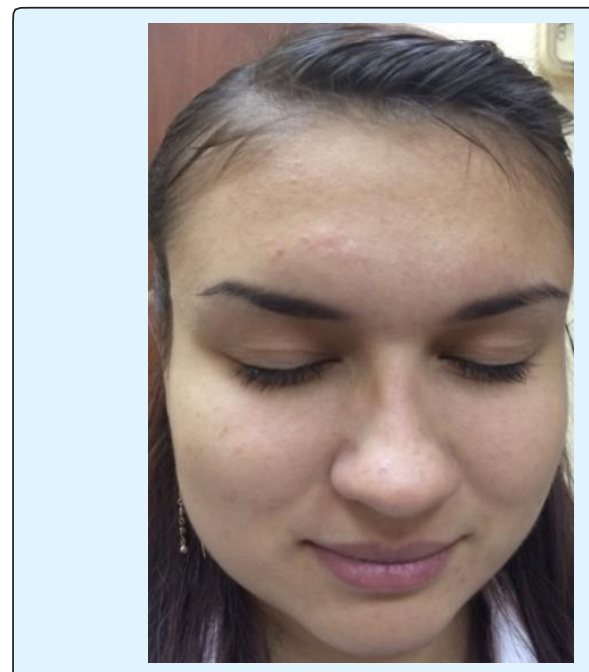

Figure 1: Patient Z., 28 years, group II. Diagnose: Rosacea, erythematotelangiectatic subtype.



Figure 3: Patient K., 35 years, group I.

Diagnose: Rosacea, papulopustules subtype.

Demodicosis. Mite load was 10 mites per $1 \mathrm{~cm}^{2}$ in scraping.

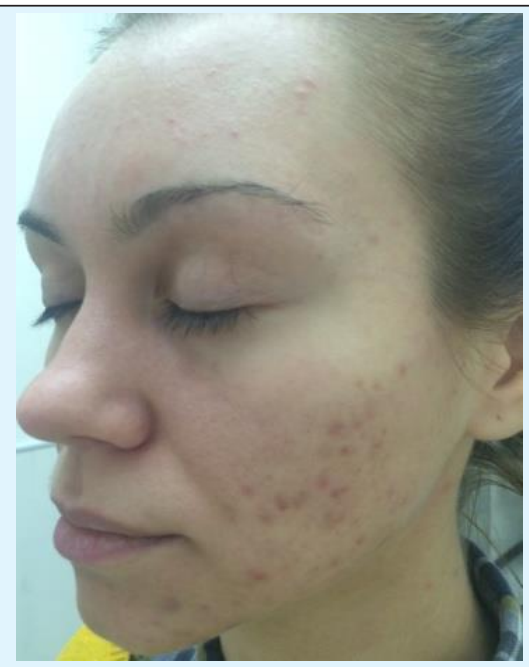

Figure 2: Patient A., 32 years, group II. Diagnose: Rosacea, papulopustules subtype.

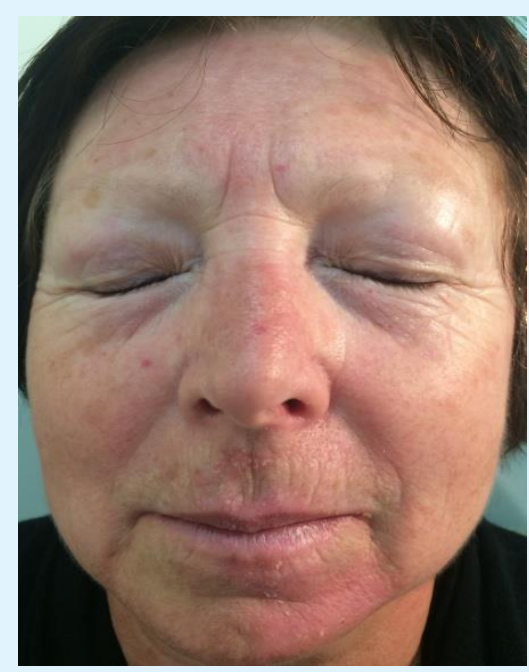

Figure 4: Patient N., 64 years, group II.

Diagnose: Rosacea, erythematotelangiectatic subtype. 


\section{Clinical Dermatology Open Access Journal}

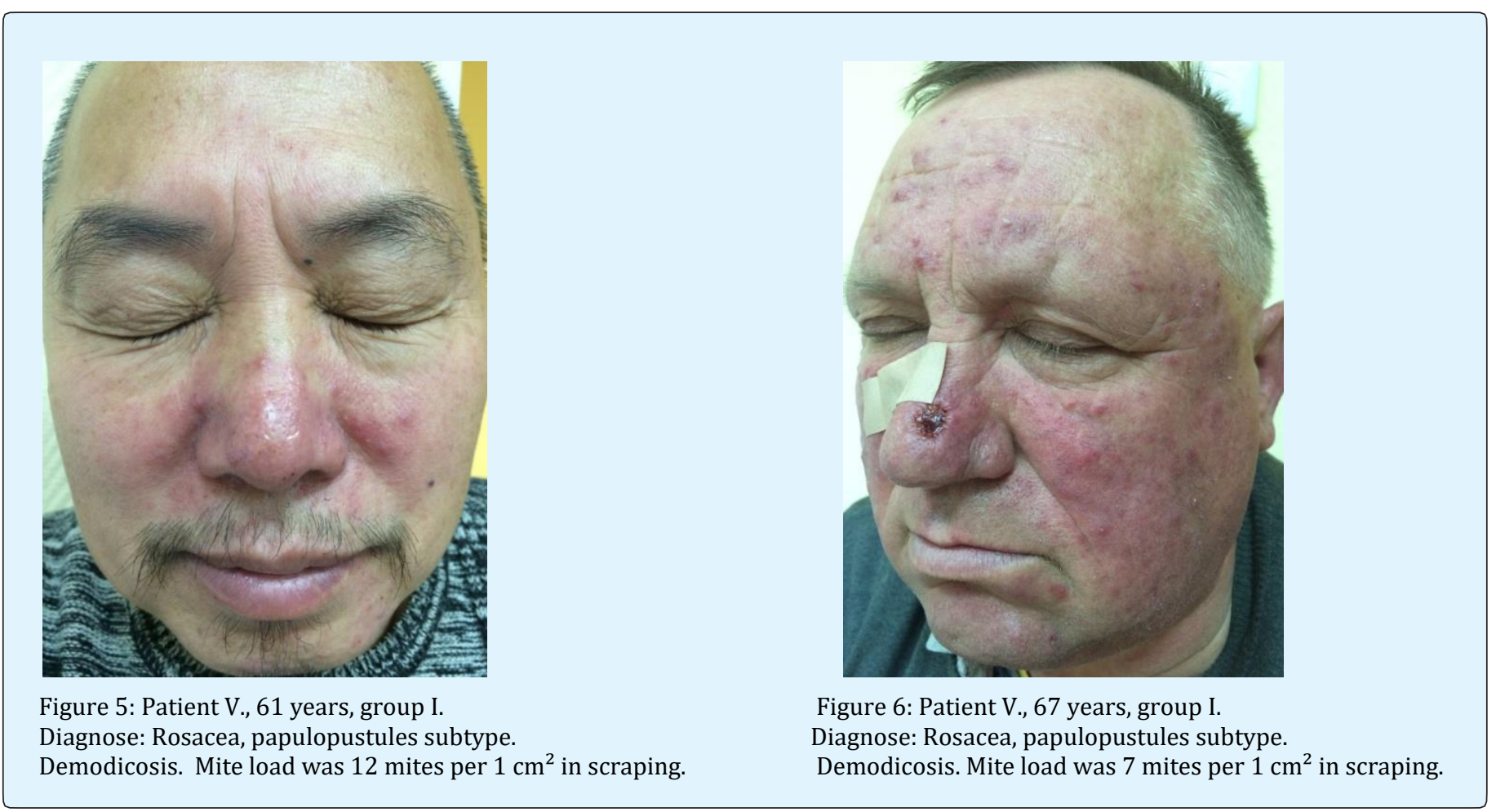

Distribution of patients according to the rosacea severity is given in table 5 .

\begin{tabular}{|c|c|c|c|}
\hline Disease form & $\begin{array}{c}\text { Group I } \\
\text { (n; \%) }\end{array}$ & $\begin{array}{c}\text { Group II } \\
\text { (n; \%) }\end{array}$ & $\begin{array}{c}\text { Total } \\
\text { (n; \%) }\end{array}$ \\
\hline $\begin{array}{c}\text { Erythematous } \\
\text { telangiectatic }\end{array}$ & $4 ; 6.7$ & $11 ; 18.3$ & $15 ; 25$ \\
\hline Papular & $5 ; 8.3$ & $14 ; 23.3$ & $19 ; 31.6$ \\
\hline Pustular & $9 ; 15^{*}$ & $5 ; 8.4$ & $14 ; 23.4$ \\
\hline Infiltrative-productive & $12 ; 20^{*}$ & 0 & $12 ; 20$ \\
\hline Total & $30 ; 50$ & $30 ; 50$ & $60 ; 100$ \\
\hline
\end{tabular}

Table 5: Distribution of patients with rosacea of groups I and II according to the degree of the disease severity.

$*_{-} \mathrm{p}=0.05$

As it is seen from the table 5, Demodex mites were detected more frequently in patients with more severe clinical forms of rosacea (pustular and infiltrativeproductive forms).

For the objectivity of the study patients with rosacea from groups I and II were divided according to the severity of the clinical manifestations of the disease (papules, pustules, open comedones, milium, telangiectasia, perifocal erythema, excoriation, pigmentation, greasy luster).
More severe clinical cases with the presence of deep papulopustules elements, nodules, perifocal erythema of the facial skin, greasy luster prevailed in group I patients $(\mathrm{p}<0.05)$ having Demodex mites. Group II patients had papules, pustules, a small amount of telangiectasia and pigmentation.

The analysis of the detection of Demodex mites on eyelashes and eye brows, has not revealed diagnostically significant excess of mites' quantity. Demodex mites were absent or were found in number of 1 individual on 2-4 eyelashes. Clinically no cases of ophthalmo-rosacea were observed.

Species identity of Demodex mites (Demodex folliculorum longus and Demodex folliculorum brevis) was determined by scraping method followed by light microscopy in patients of group I.

The largest number of cases of rosacea in group I patients was associated with parasitism of Demodex folliculorum longus ( $\mathrm{n}=20 ; 66.7 \%)$, Demodex folliculorum brevis was detected in 8 patients $(26.7 \%)^{\prime}$ both species of mites were found in 2 patients (in $6.6 \%$ of cases). Demodex folliculorum longus is significantly more often detected in patients than simultaneous parasitism of two species of mites $(\mathrm{p}<0.01)$. 


\section{Clinical Dermatology Open Access Journal}

At examining 30 healthy persons by scraping method and light microscopy, mites of the Demodex genus have been revealed in three cases $(n=3 ; 2.8 \%)$, in other 33 cases $(97.2 \%)$ detection of mites failed. The method of scraping has revealed parasitizing of Demodex folliculorum brevis in healthy persons in all cases. This suggests that in the absence of clinical picture of rosacea significantly more important than Demodex mites will not be detected in scrapings $(\mathrm{p}<0.01)$. In determining the species identity of Demodex mites in healthy individuals, the parasitism of Demodex folliculorum brevis was revealed in all three cases. People with the absence of Demodex mites in scraping and the absence of clinical manifestations of rosacea accounted for group III $(n=30)$.

Demodex mites accompanied all cases of rosacea in patients of group I. Distribution of their species identity according to diagnoses is given in table 6 .

\begin{tabular}{|c|c|}
\hline Species accessory of Demodex mites & Total (n; \%) \\
\hline Demodex folliculorum longus & $20 ; 66.7$ \\
\hline Demodex folliculorum brevis & $8 ; 26.7$ \\
\hline $\begin{array}{c}\text { Demodex folliculorum longus + Demodex } \\
\text { folliculorum brevis }\end{array}$ & $2 ; 6.6$ \\
\hline Total & $30 ; 100$ \\
\hline
\end{tabular}

Table 6: The distribution of species identity of Demodex mites among patients with rosacea, comprising the study group I.

$*-p<0.05$

Thus, in the structure of rosacea diagnoses Demodex folliculorum longus is significantly more prevalent $(\mathrm{n}=20$; $66.7 \%$ ). The quantity of cases of detecting this type of mite prevails over the frequency of detection of Demodex folliculorum brevis and the combined parasitism by two species of mites.

On the initial admission, all patients with rosacea of group I were divided by the degree of symptoms severity according to the clinical classification. Among these patients, influence of the species identity of Demodex mites has been studied.

Table 7 gives the species distribution of Demodex mites in patients of group I suffering from rosacea.

\begin{tabular}{|c|c|c|c|c|c|}
\hline $\begin{array}{c}\text { Species } \\
\text { accessory } \\
\text { of } \\
\text { Demodex } \\
\text { mites }\end{array}$ & $\begin{array}{l}\text { Erythemat } \\
\text { ous } \\
\text { telangiect } \\
\text { atic form } \\
\text { (n; \%) }\end{array}$ & $\begin{array}{c}\text { Papula } \\
r \\
\text { form } \\
(n ; \%)\end{array}$ & $\begin{array}{c}\text { Pustule } \\
\text { s form } \\
\text { (n; \%) }\end{array}$ & $\begin{array}{c}\text { Infiltrativ } \\
\text { e- } \\
\text { productiv } \\
\text { e form } \\
\text { (n; \%) }\end{array}$ & $\begin{array}{c}\text { Tota } \\
\text { l } \\
(\mathbf{n} ; \\
\%)\end{array}$ \\
\hline $\begin{array}{c}\text { Demodex } \\
\text { folliculoru } \\
\text { m longus }\end{array}$ & $0 ; 0$ & $\begin{array}{c}1 ; \\
3.3^{*}\end{array}$ & $\begin{array}{c}8 ; \\
26.7^{*}\end{array}$ & $11 ; 36.7^{*}$ & $\begin{array}{c}20 \\
66 . \\
7 \\
\end{array}$ \\
\hline $\begin{array}{c}\text { Demodex } \\
\text { folliculoru } \\
\text { m brevis }\end{array}$ & $4 ; 13.4^{*}$ & $\begin{array}{c}4 ; 13.3 \\
*\end{array}$ & - & - & $\begin{array}{c}8 ; \\
26 . \\
7 \\
\end{array}$ \\
\hline $\begin{array}{c}\text { Demodex } \\
\text { folliculoru } \\
\text { m longus } \\
+ \\
\text { Demodex } \\
\text { folliculoru } \\
\text { m brevis }\end{array}$ & - & - & $1 ; 3.3^{*}$ & $1 ; 3.3^{*}$ & $\begin{array}{l}2 ; \\
6.6\end{array}$ \\
\hline Total & $4 ; 13.4^{*}$ & $\begin{array}{c}5 ; \\
16.6 \\
\end{array}$ & $9 ; 30.0$ & $12 ; 40.0$ & $\begin{array}{c}30 ; \\
100\end{array}$ \\
\hline
\end{tabular}

Table 7: Species distribution of Demodex mites among group I patients with rosacea.

$*_{\text {- }} \mathrm{p}<0.01$

While analyzing the obtained data, it was found that Demodex folliculorum longus was detectable in all forms of rosacea, but significantly prevailed in the infiltrativeproductive form of the disease $(\mathrm{n}=11 ; 36.7 \%)(\mathrm{p}<0.01)$. Demodex folliculorum brevis was detected in mild forms of rosacea (erythematous-telangiectatic and papular) $(n=8$; $26.7 \%$ ). Two combined cases of mite parasitism have also been observed in more severe cases (pustular and infiltrative-productive forms) $(n=2 ; 6.6 \%)$.

The analysis of the disease duration in group I patients, depending on species of Demodex mites (Demodex folliculorum longus and Demodex folliculorum brevis) revealed data shown in table 8:

\begin{tabular}{|c|c|c|c|c|}
\hline Disease duration & $\begin{array}{c}\text { Demodex folliculorum } \\
\text { longus (n; \%) }\end{array}$ & $\begin{array}{c}\text { Demodex folliculorum } \\
\text { brevis (n; \%) }\end{array}$ & $\begin{array}{c}\text { Demodex folliculorum longus + } \\
\text { Demodex folliculorum brevis (n; \%) }\end{array}$ & $\begin{array}{c}\text { Total } \\
\text { (n; \%) }\end{array}$ \\
\hline Less than 1 year & $2 ; 6.7$ & $6 ; 20.0$ & - & $8 ; 26.7$ \\
\hline 1 - 5 years & $5 ; 16.7 *$ & $2 ; 6.7$ & $1 ; 3.3$ & $8 ; 26.7$ \\
\hline More than 5 years & $13 ; 43.3 *$ & - & $1 ; 3.3$ & $14 ; 46.6$ \\
\hline Total & $20 ; 66.7$ & $8 ; 26.7$ & $2 ; 6.6$ & $30 ; 100$ \\
\hline
\end{tabular}

Table 8: Duration of rosacea in patients of group I, depending on species identity of Demodex mites.

$*$ - $\mathrm{p} \leq 0.05$ 


\section{Clinical Dermatology Open Access Journal}

According to table 8, it was found that mites of Demodex folliculorum longus complicate statistically more frequently rosacea in patients of group I, resulting in disease course duration of about 5 years in comparison with Demodex folliculorum brevis and combined parasitizing of two species of mites ( $\mathrm{p} \leq 0.05)$. Rosacea, accompanied by parasitism of Demodex folliculorum brevis, was less lengthy and ranged from several months to one year ( $\mathrm{p} \leq 0.05)$, which was statistically significantly lower than in the cases associated with Demodex folliculorum longus.

\section{Discussion}

Statistically significant differences between groups in terms of gender and age have not been established.

The comparison of the frequency of rosacea recurrence between patients of groups I and II revealed a statistically significant difference $(\mathrm{p}<0.05)$. In most cases, the disease recurred only once in patients without Demodex mites. Thus, Demodex mites complicating the clinical picture of the disease, promote a more frequent recurrence of the disease.

It is known that skin diseases have a direct impact on the quality of patients' life. Practically all aspects of human life may suffer for this reason. Often, skin diseases cause serious psychological disturbance for patients, making it difficult to work in a team, communicate with friends and colleagues, perform public activities and personal relationships. Rosacea is not a life-threatening disease, but its availability has a significant impact on the patient's self-perception as an individual and adequate adaptation in the society. Patients with rosacea often undertake suicide attempts and are prone to depression and psycho-neurotic disorders. The question of influence of Demodex mites on the quality of patients' life is acute. Considering patients with rosacea, the authors used the Dermatology Life Quality Index (DLQI) as the most objective questionnaire.

Thus, according to the Dermatology Life Quality Index (DLQI) questionnaire, the presence of Demodex mites reduces the life quality of patients with rosacea. In this connection, the diagnosis of the presence of Demodex mites and the subsequent antiparasitic therapy are critical for successful therapy of the disease.

When comparing the primary and secondary morphological elements, the statistical difference between groups I and II was identified. Such abnormal formations as deep papulopustules elements, nodules, facial skin erythema, greasy luster are more pronounced in group I patients, which once again confirms that the presence of Demodex mites contributes to the emergence of inflammatory elements and promotes the development of more severe clinical forms of rosacea.

Thus, it was found that Demodex mites have a direct impact on the clinical picture of rosacea. Moreover, the severity of clinical manifestations directly correlates with species identity of Demodex mites. A longer type of species (Demodex folliculorum longus) has a higher prevalence among patients. Detection of Demodex mites in the more severe forms of rosacea (infiltrativeproductive form) gives reason to assert that the mite burdens clinical manifestations of rosacea, causing the development of papules, pustules, and nodes. In addition, while observing patients with combined cases of lesion by two types of mites (Demodex folliculorum longus and Demodex folliculorum brevis), it was found that Demodex folliculorum longus plays an important role in the development of severe clinical forms of the disease with a prolonged course.

Detection of Demodex folliculorum brevis with mild degrees of rosacea (erythematous-telangiectatic and papular form of rosacea $n=8 ; 26.7 \%$ ), and its presence in healthy individuals $(\mathrm{n}=3,2.8 \%)$ suggests that Demodex folliculorum brevis has no effect on the clinical picture of rosacea. This indicates that Demodex folliculorum brevis is presumably a saprophyte of the facial skin and patients do not need any antiparasitic therapy when it is detected.

\section{Conclusion}

In the course of the study, it was found that in the patients suffering from rosacea complicated with demodicosis, the presence of Demodex mites reduces the life quality of patients with rosacea, affecting strongly the life of patients.

Analysis of the presence of primary and secondary morphological elements in patients with rosacea showed a statistical difference between the I and II observation groups. The presence of Demodex mites promotes the development of acute inflammatory elements (deep papulopustules elements, nodes), the presence of perifocal erythema of the facial skin and greasy luster and prolongs the duration of the disease with a tendency to recur. When comparing species identity, the species of mite of Demodex folliculorum longus complicate the clinical picture of rosacea, accompanying more severe clinical forms (papular and pustular forms of rosacea). Therefore, to determine the further tactics of the therapy, 


\section{Clinical Dermatology Open Access Journal}

all patients with rosacea need to undergo an analysis for the detection of Demodex mites; and all patients with diagnoses of rosacea who have the confirmed presence of Demodex folliculorum longus in scrapings, regardless of its quantitative load, need antiparasitic therapy. At the same time, in cases of detection of Demodex folliculorum brevis, patients with rosacea do not need any specific antiparasitic therapy, since this type of mite was found in patients with mild forms of diseases and in healthy individuals and it is concluded to be a saprophyte for the facial skin.

From this perspective, it is essential to diagnose the presence of Demodex mites in all patients suffering from rosacea with mandatory identification of their species identity.

\section{References}

1. Berg M, Liden S (1989) An epidemiological Study of rosacea. Acta Derm Venereol 69(5): 419-23.

2. Baima B, Sticherlin M (2002) Demodicidosis revisited. ActaDerm Venereol 82(1): 3-6.

3. Chen W, Plewig G (2014) Human demodicosis: revisit and a proposed classification. Br. J. Dermatol 170(6): 1219-1225.

4. Akbulatova LK (1963) The pathogenic role of Demodex mite and the clinical form of demodicosis in man. Vestn Dermatol Venerol 40(12): 57-61.

5. Lacey N, Kavanagh K, Tseng SC (2009) Under the lash: Demodex mites in human diseases. Biochem (Lond) 31(4): 2-6.
6. Gutierrez Y (2000) Diagnostic Pathology of Parasitic Infections with Clinical Correlations. $2^{\text {nd }}(E d n)$, Oxford University Press, New York.

7. Ozdemir MH, Aksoy U, Sonmez E, Akisu C, Yorulmaz C, et al. (2005) Prevalence of Demodex in health personnel working in the autopsy room. Am J Forensic Med Pathol 26(1): 18-23.

8. Bohdanowicz D, Raszeja-Kotelba B (2001) Demodex in the pathogenesis of certain skin diseases. Post Dermatol Alergol 18: 51-53.

9. Whitfeld M, Gunasingam N, Leow LJ, Shirato K, Preda V (2011) Staphylococcus epidermidis: a possible role in the pustules of rosacea. J Am Acad Dermatol 64(1): 49-52.

10. Wilkin J, Dahl M, Detmar M, Drake L, Feinstein A, et al. (2002) Standard classification of rosacea: Report of the National Rosacea Society Expert Committee on the Classification and Staging of Rosacea. J Am Acad Dermatol 46(4): 584-587.

11. Finlay AY, Khan GK (1993) The Dermatology Life Quality Index: A simple practical measure for routine clinical use. British Association of Dermatologists Annual Meeting, Oxford, July 1993. British Journal of Dermatology 129(42): 27. 